\title{
Selling You and Your Clicks: Examining the Audience Commodification of Google
}

\author{
Hyunjin Kang ${ }^{1}$ and Matthew P. McAllister ${ }^{2}$ \\ ${ }^{1}$ College of Communications, The Pennsylvania State University, huk142@psu.edu \\ ${ }^{2}$ Department of Film/Video \& Media Studies, College of Communications, The Pennsylvania State Univer- \\ sity, mattmc@psu.edu
}

\begin{abstract}
Using a political economy perspective, this paper argues that Google's surplus value from advertising results from its extensive and transformative commodification of users. The unique features of Google as an advertising venue intensify the commodification of its users as compared to traditional media in several ways. First, the simplified valorization process of Google's advertising methods enables Google to earn significantly more surplus value than those of traditional media. Also, Google's personalized advertising strategies, its precise measurement of advertising costs based on users' behaviors represented as "the number of clicks," and the unclear distinction between advertisements and serviced content commodify its users' online activities. Lastly, this essay asserts that Google commodifies the aggregated consciousnesses of its users because it sells keywords for advertisers to attach advertisements to search-data output, which are priced in accordance with their popularity. This "click-through" form of behavioral commodification has significant implications for the future of media.
\end{abstract}

Keywords: Audience commodification, Political Economy of Media, Google, AdSense, AdWords

Maurice Saatchi, the well-known advertising guru, once argued that a business's brand would only be successful when a particular word comes to be associated with the business in the public's imagination. In this sense, the huge success of Google can be understood as its symbolic ownership of the word "search" in the English language (Dai, 2007). As this illustrates, Google became a major phenomenon of the new media era. People often use the word "google" as a generic verb for online searching, a practice that was signaled by the addition of this term in the Merriam-Webster Collegiate Dictionary and the Oxford English Dictionary to depict the act of Internet keyword searching (Bylund, 2006; Harris, 2006). Google explains that the origin of its company's name is related to the word "googol," which means $10^{100}$, thereby connoting the meaning of infinite digital information in cyberspace. As the company itself highlights, Google has a supercomputer network that chooses what is to be displayed in response to a search query, evaluating more than five million variables in just milliseconds (Google, 2011).

Google was founded in the late 1990s by Larry Page and Sergey Brin, two Stanford University graduate students in California. They developed their online search engine using an innovative search algorithm. In addition to the search engine, Google started to offer a variety of free applications for its users and thus established Google as the dominant new media brand. Google news, launched in 2002, aggregates and displays news from more than 4000 news sources all around the world, and is joined by Google map, Google reader, Google talk, and Google analytic. In 2006, Google acquired YouTube for $\$ 1.65$ billion in a stock-for-stock transaction (Google, 2006). In addition, Google has successfully entered to mobile service market by introducing Android, mobile operating system in 2007.

Those services are provided to its users seemingly for free. Most of Google's revenue comes from advertising not from the direct selling of its online services or applications (Google, 2011). Google's influence on new media financing, in fact, results not just from the scope of its branded applications, but also from the way it has shaped digital advertising through its lesser-known subsidiaries and advertising practices. In 2000, Google launched AdWords, which offers personalized online ad strategies for marketers, with just an initial 350 customers. Nine years later, Google (2010) reported that $96.7 \%$ of its total revenue came from advertising. With its 2007 acquisition of 
DoubleClick, an online advertising company, Google's revenue outperformed what analysts estimated even in the economic downturn (Guynn, 2008). Currently, Google has half of the U.S. market share in the Internet search market, with total revenue reaching $\$ 236.5$ billion in 2009 (Google, 2010). According to Advertising Age, Google's revenue in 2009 topped all digital-media companies and was 13th among the 100 top media companies (Johnson, 2010). Google's size, multi-task applications, and influence on web financing has changed how advertisers, media industry players -- and media scholars -- view the relationship between audiences, advertisers and media technology.

One especially apt perspective for understanding Google's altering of the media landscape is Smythe's (1981) audience commodification model. This model argues that media audiences who watch advertising while they consume media content actually work for advertisers by watching the media -- and especially the advertising -- during their supposed free "leisure" time. This perspective argues that the labor of ad-supported media audiences become commodified -- through such measurement techniques as Nielsen ratings -- and sold to advertisers. However, given what seems to be a very different economic model for new media, what precisely is the commodity (or commodities) is complicated and controversial; in addition, the traditional distinction between producers and users becomes blurred in digital media such as the Internet (McQuail, 2010). On the one hand this later point seems to grant a certain power and activity to the audience, but on the other it builds in nearly instanteous feedback mechanisms that enhance the "labor" capacity of media audiences, not just as content creators but also as data sources about their media consumption. The essay, therefore, analyzes the logics of Google's advertising system, how it commodifies and sells audiences to advertisers as illustrated by this audience commodification model. Following research questions were proposed to guide our analysis:

RQ1: How might the process of Google audience commodification be understood?

RQ2: Is user commodification model of Google more intense than audience commodification model of traditional media? If so, in what ways?

To answer the proposed research questions, the first section of this paper reviews the literature related to audience commodification in traditional media, such as television and newspapers, and how scholars have examined audience commodification in other new media forms. In addition, the study examines the advertising services of Google, such as AdWords and AdSense. Based on these analyses, the essay will dissect the mechanism of audience commodification via Google and identify the specific components of this commodification process.

\section{Audience Commodity of Traditional Media}

Commodification is "the process of turning use values into exchange values" (Mosco, 2009, p. 129). Use, as defined by individual or social needs, becomes monetized in a capitalist system, which enables the commodity to be sold in the marketplace (Mosco, 2009). Marx (1976) emphasized the distinction between the exchange value and the use value of the commodity. He explains that "the utility of a thing makes it a use-value" (p. 126). The 'exchange-value' of a commodity is determined by the abstraction of its use-value so that it can be compared with other commodities and exchanged on the open market (Marx, 1976). A modern capitalist may view this transformation as "monetization," where a usable good becomes a monetized and economically exchangeable commodity. A capitalist society can commodify just about everything, especially human labor. Surplus value -- or profit -- can be greatly enhanced when in the commodification process the exchange value of labor for the laborers is much less than the exchange value of labor for the capitalist.

Commodities, in fact, become reified in capitalism, appearing "as autonomous figures endowed with a life of their own, which enter into relations both with each other and with the human race" ( $p$. 165). Related to this Marxist notion, Mosco (2009) explained that commodities also become fetishized, resulting in a symbolic power potentially becoming even greater than religion because of "a double mystification" (p. 144): the commodity makes the socially defined relationship between capital and labor appear to be natural (that is, the commodity becomes separated from the actual 
means of production), and the commodity itself takes on its own "life," which governs the social sphere, such as in the case of packaging and the symbolism in advertising (Mosco, 2009).

Garnham (1979) elaborated on the process of media commodification in modern society. He argued that there are two principle aspects of media commodification. Media products (i.e., media content) can directly become commodities (movie ticket sales and DVDs, for example). However, a second commodification process is the focus of this essay. He argued that audiences are the most important commodity of in advertising-supported mass media. Smythe (1981) also suggested that audiences not only buy commodities and services but are also produced and sold as commodities through mass media to advertisers. Media companies produce content that attract audiences and encourage, trick, or seduce viewers to watch the accompanying advertisements. Consequently, the cost that companies pay for advertising spots is ultimately used for buying the audiences that watch the spots.

Therefore, it can be argued that audiences are also a form of commodified labor on television (and other ad-supported media) in modern capitalist societies. Smythe (1981) asserted that, aside from sleeping, every hour for an individual is "unfree work time" (p. 248). Jhally and Livant (1986) developed this "audience-as-labor" concept, arguing that "watching as working' is both a real economic process, a value-creating process, and a metaphor, a reflection of value creation in the economy as a whole" (p. 125). By using the metaphor of "working" for watching television, they articulated the process by which leisure time can be used to regulate the demand of goods, thus reducing the costs of storage and distribution while simultaneously "paying" audiences with supposedly free media content that is often commodity-friendly itself but seems autonomous from the advertising. Additionally, because advertisers are the "customers" for this "audience-product labor," the ad-supported media industries' logic is such that the products these customers find most desirable are attracted, sought out, and sold to the advertiser. Some audience commodities, then, are more valuable than others according to the audience's socio-economic status and their likelihood of consuming these products and being persuaded. In this way, Smythe broadened the narrow concepts of capitalist "production" by incorporating distribution and consumption processes in a service-oriented economy.

On the other hand, Mosco (2009) clarified the meaning of commodification by comparing it with commercialization and objectification. According to him, commodification and objectification are broader terms than commercialization. Commercialization mainly refers to the relationship between an advertiser and the audiences who that advertiser targets and the integration of advertising and promotional logic into different aspects of society. Clearly, then, audience commodification is strongly related to commercialization, but commodification itself may involve other forms of commodification besides those found in an advertising-based system. However, audience commodification concerns of the treatment of humans as reified objects; thus, it is a form of audience objectification in broader processes, such as the commodification of labor (Mosco, 2009). How the industry constructs audiences through bottom-line characteristics that are valuable to economic interests-characterizations of audience size and demographic categories, for example-illustrates both the objectification of viewers as well as that process's economic logic.

Given the dependence on the commodified-labor audience for the economics of television, how the audiences are measured becomes critical. Meehan (1984) proposed the central role of "ratings" in the process of audience commodification. She argued that the "something" that is exchanged by the process of audience commodification is neither the media messages nor the audiences themselves, but "ratings." Audience ratings, which have traditionally been conducted by consumer research companies, such as A.C. Nielsen, report the characteristics of media audiences, including size, demographic patterns, and media-usage patterns. What Meehan (1984) claimed was that rating information is exchanged by media companies and advertisers and that ratings are, therefore, the primary commodity of the media, not the audiences themselves. Of course, Nielsen uses various sampling methods to acquire ratings data, and these methods are then extrapolated to what is assumed to be larger audience-viewing patterns, including the watching of specific programs and the commercials within those programs. In fact, because this information is so crucial to 
the day-to-day activities of ad-supported media and its measurements are contested if collected by self-interested parties, companies like Nielsen have been able to function as monopolies, providing one set of commodified data to which both media and advertisers may subscribe (Meehan, 2005). Nevertheless, these important audience-commodity measurements for television and radio continue to be a contentious area as competition for audiences increase and digitization offers additional measurement criteria.

Mosco (2009) explained that one reason Meehan's idea of ratings as the center of media commodities is valuable is because it highlights the issue of the "cybernetic nature of the commodification process" ( $p .150)$. Audience ratings function to monitor the process of commodification and serve as a measurement necessary for commodity production. In this sense, ratings are a critical part of commodification because this information is used in making media content attractive to the target audiences (such as promotions for programs that assert "The Number One Show in America!") and, consequently, help media create additional audience commodities.

Based on the literature, there are at least five organizations or groups of people involved in the "cybernetic" process of traditional ad-supported media audience commodification: advertisers, advertising agencies, consumer research companies, media, and audiences. These groups are organically connected to each other by each group's industrialized necessities, and these connections eventually function to commodify media audiences. Advertisers buy advertising services from advertising agencies, and they buy consumer information from consumer research companies. Audiences, in turn, watch "free" media content as well as advertisements delivered along with them, and some of those who are persuaded by the advertisements eventually buy the advertised products. Those audiences measured in the current ratings system have their demographic and behavioral data transmitted to consumer research companies, which then processes this data as meaningful information-namely ratings-which is sold to advertisers and media. This audience commodification process can be visually depicted, as in figure 1 .

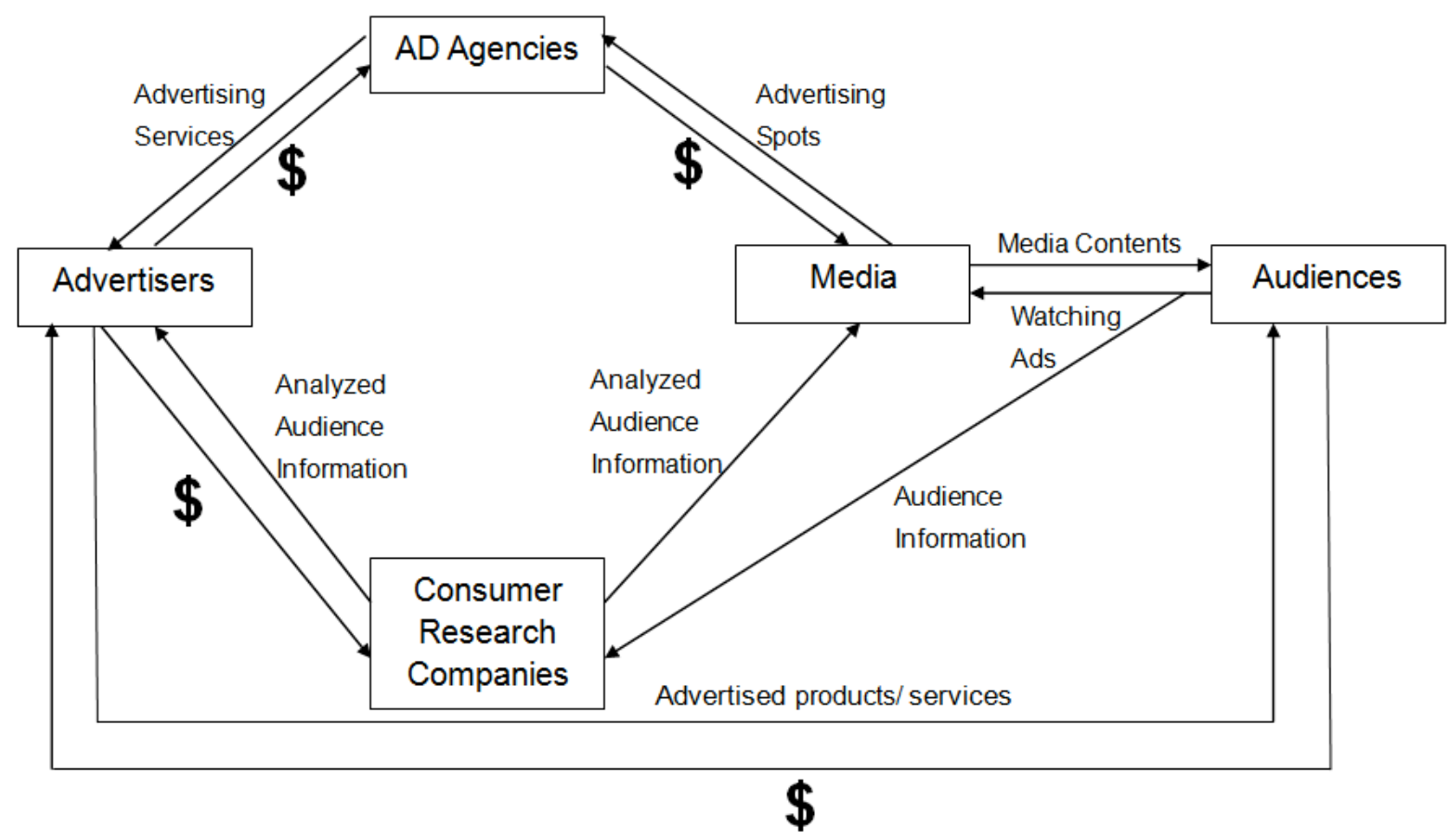

Figure 1: Process of traditional media audience commodification 


\section{Audience Commodity of New Media}

Thanks to the rapid advancement of media technologies, media audiences now can enjoy various kinds of media content and forms. However, using the notion of political economy, media scholars (e.g., Andrejevic, 2006, 2009; Cohen, 2008; Terranova, 2000; van Dijck, 2009) argue that today's most popular websites, including Facebook, YouTube, and Google, lead to the extensive commodification of their users in diverse ways.

Recent interactive media technologies, such as remote controls, digital video recorders (DVR), and the Internet, seemed to have transformed media "audiences" into "users" who can control and even produce the media content they consume to a greater degree than ever before. For example, thanks to these technologies, media users no longer have to passively view the advertisements delivered to them along with media content. Therefore, some suggest that these new media technologies challenge the forced advertisement-viewing assumptions of TV (as reviewed by Carlson, 2006), which was considered to be the basic premise of "labor" under the political economy perspective. Ironically enough, however, the more agency media technologies render to users, the more intensified and diversified is the free labor that users provide to media industries. In other words, passive advertisement viewing is not the only "commodifiable labor" in modern media industries because the interactive nature of new media makes it possible to commodify various user activities, especially the information users produce by such activities.

One notable difference of Web 2.0 from that of traditional media (e.g., television) is that the distinction between producers and consumers has become obscured. There is no clear cut between what is being produced and sold on the Internet (Bermejo, 2009). This led to recent controversial debate on whether or not Smythe (1981)'s audience commodification model can be equally applied to the new media environment (McQuail, 2010). Unlike most traditional media, Web 2.0 largely depends on content, information, and even networks that are created and uploaded by users. For example, media audiences voluntarily produce and distribute self-created media content via YouTube, actively input personal information on their MySpace profiles, and construct and display their social networks on Facebook. Such voluntary and excessive activity performed on the Internet not only generates sellable personal information but also cultural content, including personal tastes and preferences, and other forms of user-generated content, from which Internet companies create capital value (Terranova, 2000) by attaching ads to this content and facilitating market research. Coté and Pybus (2007) defined such free labor in which "subjects engage in on a cultural and biopolitical level" (p. 90) in social networking sites as "immaterial labour 2.0." Obviously, in this case, user labor enters the system much earlier than in traditional media. Because users of social networking site are producers and consumers of cultural components that such websites provide, it can be said that their labor in Web 2.0 begins during, not after, the production and broadcast of media content (Cohen, 2008).

The interactive nature of the Internet not only requires and encourages users to provide extensive labor for creative activities, but it also facilitates surveillance-the fundamental mechanism of audience commodification in new media. As mentioned before, interactive websites commodify socio-cultural components that users voluntarily create and input in almost every activity on social network sites. Therefore, commercially oriented surveillance of user activity on websites is the most crucial and fundamental mechanism that enables companies to establish profitable business models. For instance, Facebook - the most popular social networking site in the United Statescommodifies its user through extensive surveillance (Cohen, 2008). According to Cohen, surveillance enables web service providers not only to aggregate but also render its users' information for third-parties so they can perform precisely targeted marketing activities.

In sum, compared to other traditional types of media, the interactive nature of the Internet both allows for and depends on its users to be engaged in extensive-and perhaps economically exploitative-uploaded activities from providing personal information to the production of content. Also, media companies create capital value out of web user activities via the extensive surveillance that is enabled by current computer technologies. That is, user data generated by interactive activities on the web are stored in mass storage devices, and then the stored data are processed to 
provide meaningful information through data mining, thereby creating revenues of such website providers.

\section{Audience Commodity of Google}

Although Google-primarily known for its influential search engine-is not commonly classified into Web 2.0, it also inculcates its users as part of the system of a commodified "extensive labour 2.0" (Coté \& Pybus, 2007). Applying ideas from the previous sections, this study argues that identical to how Web 2.0 intensifies audience-labor commodification, Google commodifies its users through extensive surveillance by utilizing interactive technologies but with a streamlined and influential model that actually intensifies the level of audience commodification.

Since the early days of industrialized media, the search engine is arguably the most important technological development for advertisers because it enables advertisers to precisely target individual consumers given their inputted interests, which may in turn be connected to advertised products or services (Spurgeon, 2008). Google has been at the center of the development of search-based advertising since its onset and is making enormous profits out of advertising associated with keyword searches. In one sense, the Google search engine exploits the collective labor of the Internet since search engines are only attractive if there is a large amount of web information to search, web information that Google did not create itself. This is what Vaidhyanathan (2011) largely meant in his phrase the "Googlization of everything"-namely, that Google works by "harvesting, copying, aggregating and ranking of information about and contributions made by each of us" (p. 83).

In addition, as in the Web 2.0 audience commodification model, online activity surveillance is the core mechanism underlying Google's advertising strategy. In fact, Google's most important business purpose is not to facilitate searches but to sell spaces for advertisement; more precisely speaking, Google sells its users' cognition, including attention (Vaidhyanathan, 2011), to the advertisements targeted to their search behaviors, an attention that, in turn, is able to be operationalized through users' measurable behaviors subsequent to their searches, as detailed below.

\subsection{Advertising Services of Google: AdWords, Adsense and DoubleClick}

Most of Google's revenues come from pay- (or cost-) per-click advertising services (Perez \& McMillan, 2007). To provide an advertising service that precisely targets users who have interests related to the advertiser's product and service, Google employs a unique ad program, named AdWords. With AdWords, search keywords entered in the Google search engine are automatically associated with targeted advertised products or services through text-based advertisements on the Google results pages. Google does not limit smart advertising execution to its owned application web pages; it also runs a global advertising program called "AdSense." Anyone who owns a website can register the website on the Google network. Using the AdWords program, Google places advertisements for external websites on websites with similar content. For example, if a registered website contains information about French recipes, you may find an ad for websites about French restaurants or cooking wares that are labeled as "Ads by Google."

The cost of the keyword-matching advertising service and the revenue from those who participate in the AdSense program are based on the keyword-bidding system. Advertisers can choose any keywords that they want to link advertisements, and bid an amount they are willing to pay for each click (i.e., cost-per-click (CPC)) on the advertisement displayed on Google or one of its partner's sites. The higher the amount of the bid, the greater the chance the advertisement appears on the first page of the search result, or on a good location of one of web-pages participating in AdSense program.

Google's advertising software scans and processes the text on the results pages of each keyword search and enables Google to target ads precisely related to the site's content and ultimately each web user's needs. Google places advertisements, which they call "sponsored links," on the upper right hand side of the search results page. Because these links are very similar to users' 
search results in both appearance and content, many users may not even realize that the space for those advertisements occupies almost one-third of the page. Moreover, Google's Gmail system is similarly coordinated so that users are exposed to targeted advertisements when they read an email. The AdWords program analyzes the texts in users' personal email messages and places advertisements that match the text. For instance, when one reads an email about a trip to Chicago, one is also provided with links that lead to websites for hotels and restaurants in Chicago. In addition, following the analysis of van Dijck (2009), YouTube, the Google-owned video-sharing site, is similarly commodified and includes the placement of targeted "Promoted Videos" at the top left of the search results.

In addition to keyword-matching ads, Google has been providing banner, graphical and video ads services since they acquired DoubleClick in 2007. DoubleClick, founded in 1996, provides services and tools to display both rich media ads and search engine-based pay-per-click ads. This acquisition seemed to be made because Google had been lagging behind other portal sites such as Yahoo in these multi-media online ads, although Google was huge successful in keywordmatching text based ads (Perez \& McMillan, 2007). Alsp, DoubleClick provides data about behaviors of users on other websites to Google by collecting and networking such data. Consequently, DoubleClick plays an important role to help Google's ads to target users precisely through extensive surveillance (Fuchs, 2011).

To maximize marketing decisions based on website data, another sub-brand, Google Analytics functions similarly to both media planning advertising agencies and the television rating system but at a much more sophisticated level. It analyzes user preferences, allows advertisers to target potential consumers whose interests match the advertised products, and automatically places ads on advertisers' websites who use Google Analytics. Unlike the traditional data offered by Nielsen, Google Analytics literally analyzes each individual user's interests-at least as manifested by web surfing - not an anonymous and constructed group of people who, based on data from a limited sample, may share similar interests or demographic characteristics. Therefore, Google's keywordmatching ads play a central role not just in Google's audience commodification but also in refining the techniques of audience commodification of media in general.

We can understand the process of commodification of Google users by examining how these programs function in relation to advertisers, Google, and its users. The process of Google's audience commodification can be described visually as in figure 2 .

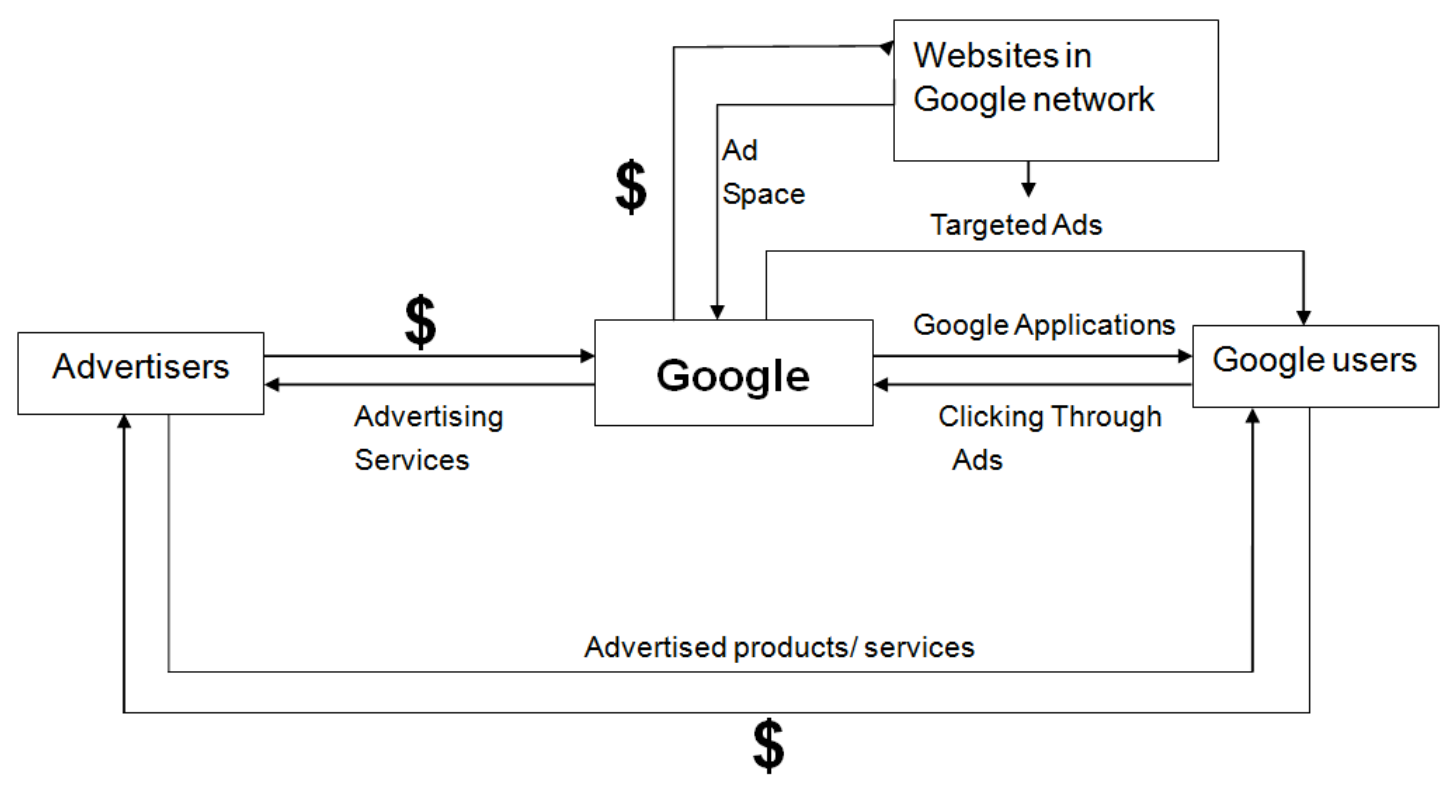

Figure 2. Process of Google's audience commodification 
As figure 1 and figure 2 illustrate, the procedure of audience commodification is streamlined for Google as compared to the television model. This more precise and, in many ways more automated, process of audience commodification increases the surplus value of advertisers. As mentioned earlier, Jhally and Livant (1986) argued that speeding up the valorization of a commodity is the key role of television commercials for creating surplus value; it makes the movement of goods much more efficient. Therefore, the efficient and extensive the process of a commodity's valorization, the more surplus advertisers can acquire because streamlined advertising processes enable advertisers to significantly decrease cost and increase commodity information. Reduced cost from the simplified procedure of commodity valorization results in creating surplus value, some of which eventually contributes to Google's revenue.

In the process of Google user commodification, we can observe that there is no need for a consumer research institution, such as A.C. Nielson. This is because Google monitors users' online behaviors (including the search terms they input) and exposes them to advertisements simultaneously. Google's advertisement programs enable advertisers to precisely target audiences who might be interested in the advertised products or services based on their behavioral measures. Google acquires strong indications of users' interests by analyzing the words they enter in Google's search engine, the email texts that they receive via their Gmail accounts, and the website content they read. Therefore, the user interests that are analyzed by Google are much more precise than television program rating systems. Rating systems analyze the demographic make-up and media usage patterns of a sample of the television audience. Based on this information, advertisers and media companies make reasonable "assumptions" about the audience's interests and even attentiveness toward the ads. Because this analysis is based upon a sample and the assumption that program viewing equals ad viewing, it cannot precisely predict the interests of individuals within a larger group, as many errors would ultimately result. However, advertisements executed via AdSense do not target groups of people but rather targets individuals and targets them because of specific individual behaviors (typed words) instead of assumed behaviors (viewing patterns based upon a Nielsen sample).

Therefore, the value of the "immaterial labor" of Google users is determined in a concrete and precise way. With traditional television ratings, several assumptions must be made: (1) the Nielsen sample accurately represents the desired audience market, (2) those watching the programs also watch the ads, (3) those watching the ads will be persuaded by them, and (4) this persuasion in the home leads to purchasing behavior. With the Google model of audience commodification, the effectiveness of an advertisement is determined by whether users click the provided link or not. Unlike traditional media, the "click through" is not a guess about whether the audiences are watching-let alone being persuaded by-the advertisement. How precisely Google adds value to its users' labor through the click-through measurement is found on Google's web page, which describes how they determine the rank of ads for display:

AdWords ranks ads for display in one of two ways: either by CPM (cost per thousand) or a by combination of the maximum cost per click (CPC), which is set by the advertiser, together with clickthrough rates and other factors used to determine the relevance of the ads. This process favors the ads that are most relevant to users, improving the experience for both the person looking for information and the advertiser looking for interested customers (Google, 2011).

As the statement reveals, to gauge the cost of advertising depends on "the number of clicks." This fact also makes additional audience research less necessary or at least adds much to the data of how audiences relate to ads. Advertisers who use traditional media as the conduit for their advertisements spend a great deal of money measuring the psychological effectiveness of advertisements, such as attitudes and behavior intentions. However, by completely restricting the measurement for an advertisement's effectiveness to behavioral consequences, measuring psychological effectiveness is largely unnecessary for Google and its advertisers. The fact that Google measures 
advertising effectiveness exclusively by behavioral factors indicates that users as commodities actually have a concrete value according to the number of times they click advertisements. This behavioral measurement does add a new complication: the danger of "click fraud" from competitors or websites looking to boost revenue (Spurgeon, 2008, pp. 31-32). However, AdWords also addresses ways to ameliorate such practices in its help literature ("How does Google detect invalid clicks," n.d.), and the potential "noise" such practices create are well within tolerance levels given the usefulness of the data.

Consequently, audience analysis, advertisement exposure, and users' reactions to the advertisement can occur in mere seconds. This extremely simplified procedure of commodity valorization saves advertisers the production costs normally associated with television commercials. Because targeted users' exposure to advertisements and website visits can occur almost simultaneously, Google and its advertisers do not need to be concerned about ad recalls or attitudes toward the advertisement itself. Therefore, advertisers do not need to invest money in creating eye-catching or memorable advertisements as much as TV advertisers do. Additionally, when a user clicks through an online ad, not only is this evidence that the user was attracted to the ad, but it typically takes the user to an online retail space where purchases can immediately follow.

As a result, virtually every Google user becomes a producer of information for Google. Google users input their interests by putting keywords in the Google search box in order to retrieve information about their interests that is available on the web. Such data are gathered by the Google system and then provides meaningful information-namely "trends" to the advertisers. Not only does this program offer the most effective "keywords, ads, referrals and campaigns" that contribute to the advertisers' revenue, but it also gives information about "where visitors come from and how they interact with your site" (Google, 2010). Google provides advertisers with much more precise information about the target audience than normal consumer research companies can provide. Also, this Google advertising system has significantly reduced the time required for a company to earn revenues from its advertisements. By providing advertisements in the form of website hyperlinks, Google makes it easy for users to visit the website immediately after they are exposed to the advertisement.

Moreover, the "click-through" model will not necessarily be limited to the Internet as other media forms, such as television, become increasingly digitized and interactive; in fact, Google has explored movements into television (Andrejevic, 2009). This expansion has implications both for changing the economic logic of television and for the "stickiness" of ad campaigns that may follow us across media platforms (Andrejevic, 2009).

\subsection{Google's Audience Commodification-Privacy, Activity, and Cognition}

Google's targeted advertisement strategy severely threatens users' privacy-the dark side of personalized advertisement enabled by technological surveillance. Google claims that its goal is to make users who are exposed to these personalized ads find them useful (Google, 2011). It certainly is possible that a large number of people would feel convenience or even self-fulfillment from the advertisements that serve their interests. However, Vaidhyanathan (2011) pointed out that Google's privacy policy actually focuses more on retaining its right to utilize user information for its own interests than protecting it. This policy even states that Google may collect IP (Internet Protocol) addresses, search histories, and Web browser settings and preferences and information (Vaidhyanathan, 2011). Although this information seems to be trivial for individual users, when they are aggregated and processed by Google, they come to have exchangeable value.

However, just as television commercials may be annoying, to what extent are users significantly annoyed/distracted by Google's advertising and data-collection practices? Are users even aware of the fact that they are being deprived of their privacy at the cost of receiving advertisements that represent their own interest? Lester (2001) criticized the business sector's indifference to the fact that new information technologies are imposing a significant threat to people's privacy. In 1999, Sun Microsystems CEO Scott McNealy rebuked the public when he said "You have no privacy, get over it" (Lester, 2001, p. 27). In modern society, it seems that people cannot function as a member 
of society without exposing private information willingly or unwillingly. They expose their information on a daily basis by exchanging emails, using credit cards, and browsing the Internet.

Andrejevic (2006) argued that the nature of new technology's interactivity is, in fact, causing a significant loss of privacy among non-equal parties. Interactivity implies a somewhat positive connotation in that it is a "two-way, symmetrical, and relatively transparent communication"; however, the strategically assimilated form of interactivity that occurs through Internet businesses are "asymmetrical, nontransparent forms of monitoring and surveillance" (Andrejevic, 2006, p. 393). Similarly, Meyrowitz (1985) cautioned that the loss of privacy in and of itself may not be as troubling as the "nonreciprocal loss of privacy" where citizens do not have "the ability to monitor those who monitor us" (p. 322). The nonreciprocal loss of privacy can easily be detected in the relationship between Google and its users. The users give their information either voluntarily or without their knowledge while using Google applications. For example, users search for information about a disease and its symptoms about which they are concerned via a Google search; they exchange summer vacation plans with friends via Gmail; they put their addresses and their travel destinations to get maps via Google Map. Yet, we do not even know the basic algorithm behind Google's search engine because this search algorithm is their top business secret.

Interactivity of the Internet is at the heart of the commodification of digital audiences. Andrejevic (2006) criticized the irony of new media's interactivity, stating that "the labor of detailed information gathering and comprehensive monitoring is being offloaded onto consumers in the name of their empowerment" (p. 393). According to Andrejevic's notion, the commodity that Google produces and sells is not only its users' private information but is also its users Internet activities, such as browsing web pages, inputting personal information, writing emails, and so on. The longer a user stays on a certain web page, the greater chance there is of that user visiting advertisers' websites through the links provided by the AdWords program. This is the reason why Google provides its users with various applications and constantly develops applications that may attract other users. Those free applications can be compared to a "free lunch," which is how Smythe (1981) metaphorically described free media content provided to audiences by media companies. However, as Smythe also implies, the free lunch in such economic exchanges is a myth; there is a cost to the viewer/user that is often hidden but sometimes made explicit. As compensation for watching advertisements and voluntarily giving their information, Google users enjoy free services, such as free Gmail accounts and instant/precise information searches.

In addition, "the number of clicks" has more value for Google than may initially seem. The more profound value that lies underneath "the number of clicks" can be found in the process that advertisers use to determine the budget for their AdWords advertising. When advertisers register their advertisement in the AdWords program, they need to choose keywords that are relevant to their own business. For instance, if an advertiser is running a coffee business, then he/she would choose keywords like coffee, coffee beans, and so on. Then, the program would determine the maximum budget for running the advertisement per day or per month and the maximum amount of money that the advertiser is willing to pay per click. This cost per click (CPC) will affect the amount of user exposure to the company's advertisement. In short, the effectiveness of advertising is determined by which keywords the advertiser chooses and the amount of CPC. If an advertiser chooses popular keywords and sets a high CPC, then the advertising cost will increase, and naturally, the advertisement's effectiveness will increase as well. The popularity of each keywordmore specifically, the degree of information popularity that people seek-is a commodity of Google. In other words, information that is searched for by many people will earn more money for Google.

\subsection{Further Erosion of Labor and Leisure Time}

However, the degree to which audiences are purposely laboring through clicking is debatable. Just as some television ads try to mimic television programs, it seems that Google imposed a wise strategy to maximize "the number of clicks." The form of advertisements found on the Google search page, which they call "sponsored links," is very similar to the form of the information that we expect to get from the Google search itself. Therefore, users who do not know the nature of a Goo- 
gle search may click the sponsored links without knowing that these links are actually advertisements. What is more, there is no distinction between the labor time and "free lunch" (Smythe, 1981, p. 243) time on Google. For example, users can have free and instant information through a sophisticated search engine and can use a free email account that offers more than 6,000 MB for each account. However, while they enjoy these free Google applications, the automated systems of Google read users email, analyze keywords that are inputted in search boxes, and give personalized advertisements. While watching traditional advertising in exchange for programming may be a fairly clear trade, the hidden nature of our commodification on Google (i.e., unavoidable ads thematically linked to content, online behavior) and the compensation (i.e., free applications) tends to deprive users of agency in this process. The commercial/program distinction that was obvious in the traditional television model is eradicated in Google's version. At least, TV viewers can switch channels during commercials. As discussed earlier, scholars such as Mosco (2009) argue that commodity fetishism occurs when the production context of a commodity becomes separated from the commodity while other cultural meanings are added (often by advertising). The commodification process of traditional advertising-supported media such as television is fetishized given that the process is generally hidden from audiences (such as Nielsen ratings measurement practices) while cultural benefits are touted (as in the miracle of "free television"). Applying this perspective, the audience commodification of digital media, as defined by Google, is even more fetishized. The interactive web services are also seemingly free but more customizable, the targeted ads are personalized and match our interests and web content as if by magic, and how our data is collected, used and monetized is automatic, instant, and largely masked.

Now it becomes clear that not only Internet users' labor (i.e., providing their information to Google and other Internet activities) but also users' aggregated consciousnesses are commodities of Google. Google provides an application called "Google Trend" with which anybody can see the search patterns of any keyword. This application shows the search patterns of keywords in the form of graphs so that users can easily get an idea of a keyword's popularity. It not only provides the popularity of keywords but also gives the periodical patterns of this popularity. As such, this application is very useful for current or potential Google advertisers. Based on this information, advertisers can plan effective advertisement strategies via Google by choosing popular keywords among those related to their product. Through Google's powerful search engine service, users' interests and consciousnesses are aggregated and reproduced into valuable information.

\section{Conclusion}

Based on the assertions of political economy scholars who discussed the commodification of audiences, this essay analyzed the process by which Google users become its commodities. Google has made the user commodification process more precise and much intensive than ever, To summarize, its audience commodification process involves:

- Google practices much more direct audience commodification than traditional media. By not relying on estimates of audience use patterns based on sampling from a third-party like Nielsen, commodification can be instant and more cost efficient

- The targeted advertising strategy of Google intensifies the commodification of audiences. Google uses behavioral information provided directly by its users of attention to advertising. Therefore, it can be said that each individual, as opposed to a speculative and constructed aggregate, is a commodity of Google.

- Google commodifies diverse components of its users, including private information, online behaviors, and cognitions. The activities through which people willingly or unwillingly give their personal data while they use the Internet and their aggregated consciousnesses (which is represented by the popularity of keywords) are also commodities of Google. Thus, it seems that Google may acquire information about users' social networks and other lifestyle and financial indicators. This will result in the commodification of social relations as well. Google's purchase 
of DoubleClick means that Google is developing banner and video ads besides search ads.

- In the commodification model of Google, there is even less distinction between leisure and labor time, given especially the multi-function and multi-entertainment nature of the Internet.

- Google's audience commodification model, if applied to digitized versions of traditional media such as television, could raise expectations about audience information collected from those media and the level of integration between advertising and media content.

These characteristics of audience commodification of Google resulted in reduced costs and enhanced revenue collection - that is, more surplus value-for Google. Using advanced technology, the cybernetic commodification of Google users is becoming sophisticated and stealthy but also wide-ranging. Because of the nature of the business, Google will continuously try to invent new strategies to increase their profit, which directly links to the commodification of its users. Given the fact that Google's growth has been achieved at the expense of the commodification of its users' personal details, consciousnesses, and social networks, users should be aware of why Google is providing various applications with no charge. Google has been playing a critical role in establishing norms of information production and sharing on the Internet. As scholars, we can understand the logics of such audience commodification models, the directions about privacy and advertising intrusiveness that such logics may take us, and the implications of such logics for our democracy and needed media policies.

Given the fact that the Internet has its potential to be an effective tool that can liberate people from centralization of information production and sharing, we can create different information sharing mechanisms that can against Google's audience logics. Creating media networks among information workers so that Internet users can acquire information they need without using commercialized Internet mechanisms can be established as, ironically, new media companies like Google become more visible, powerful, and thus spark user backlash in a system touted as empowering.

\section{References}

Andrejevic, M. (2006). The Discipline of Watching: Detection, Risk, and Lateral Surveillance. Critical Studies in Media Communication, 23(5), 391-407.

Andrejevic, M. (2009). The Twenty-First-Century Telescreen. In G. Turner \& J. Tay (Eds.), Television Studies after TV: Understanding Television in the Post-Broadcast Era (pp. 31-40). New York: Routledge.

Bermejo, F. (2009). Audience Manufacture in Historical Perspective: From Broadcasting to Google. New Media \& Society, 11(1-2), 133-154. doi: 10.1177/1461444808099579.

Bylund, A. (2006, July 5). To Google or not to Google. The Motley Fool. Retrieved from: http://www.fool.com/investing/dividends-income/2006/07/05/to-google-or-not-to-google.aspx

Carlson, M. (2006). Tapping into TiVo: Digital Video Recorders and the Transition from Schedules to Surveillance in Television. New Media \& Society, 6(1), 97-116.

Cohen, N. S. (2008). The Valorization of Surveillance: Towards a Political Economy of Facebook. Democratic Communiqué, 22(1), 5-21.

Coté, M. \& Pybus, J. (2007). Learning to Immaterial Labour 2.0: MySpace and Social Networks. Ephemera, 7(1), 88-106.

Dai, X. (2007). Google. New Political Economy, 12(3), 432-442.

Fuchs, C. (2011). Web 2.0 Prosumption, and Surveillance. Surveillance \& Society, 8(3), 288-309.

Garnham, N. (1979). Contribution to a Political Economy of Mass Communication. Media, Culture and Society, 1(2), 123146.

Google (2006). Google to Acquire YouTube for \$1.65 Billion in Stock. Google Press. Retrieved from http://www.google.com/intl/en/press/pressrel/google_youtube.html

Google (2010). 2010 Financial Tables. Google Investor Relations. Retrieved from http://investor.google.com/financial/tables.html.

Google (2011). Product Descriptions. Google Press. Retrieved from http://www.google.com/intl/en/about/corporate/company/

Guynn, J. (2008, April 18). Google's Earnings jump 30\%. Los Angeles Times, C.1. 
Harris, S. D. (2006, July 7). Dictionary Adds Verb: To Google. San Jose Mercury News. Retrieved from http://adwords.google.com/support/aw/bin/answer.py?hl=en\&answer=6114.

Jhally, S., \& Livant, B. (1986). The Television Audience Watching: The Valorization of Audience Consciousness. Journal of Communication, 36(3), 124-134.

Johnson, B. (2010). 100 Leading Media Companies. Advertising Age, Retrived from http://adage.com/mediaworks/article?article_id=146004.

Lester, T. (2001). The Reinvention of Privacy. The Atlantic Monthly, 287(3), 27-39.

Marx, K. (1976). Capital: A Critique of Political Economy (Vol. 1). (B. Fowkes, Trans.) London: Penguin (orig. 1867).

McQuail, D. (2010). McQuail's Mass Communication Theory (6th ed.). London: Sage.

Meehan, E. R. (1984). Ratings and the Institutional Approach: A third answer to the Commodity Question. Critical Studies in Mass Communication, 1(2), 216-225.

Meehan, E. R. (2005). Why TV is Not Our Fault: Television Programming, Viewers, and Who's Really in Charge. Lanham, MD: Rowman \& Littlefield.

Meyrowitz, J. (1985). No Sense of Place. New York: Oxford.

Mosco, V. (2009). The Political Economy of Communication (2nd Ed). London: SAGE Publications.

Perez, J. C., \& McMillan, R. (2007). Google to Buy DoubleClick for \$3.1 Billion. PC World. Retrived from http://www.pcworld.com/article/130722/article.html.

Smythe, D. W. (1981). On the Audience Commodity and its Work. In Dependency Road: Communications, Capitalism, Consciousness, and Canada (pp. 22-51). Norwood, NJ: Alblex.

Spurgeon, C. (2008). Advertising and New Media. New York: Routledge.

Terranova, T. (2000). Free Labor: Producing Culture for the Digital Economy. Social Text, 18(2), 33-58.

Vaidhyanathan, S. (2011). The Googlization of Everything (And Why We Should Worry. University of California Press.

Van Dijck, J. (2009). Users Like You? Theorizing Agency in User-Generated Content. Media, Culture \& Society, 31(1), 4158.

\section{About the Authors}

Hyunjin Kang

is currently working towards a Ph.D. in Mass Communications at The Pennsylvania State University. She earned M.A. in Public Relations (2005) from Michigan State University, and B.A.s in Advertising and Public Relations (2002) and in Business Administrations (2002) from Ewha Womans University in Seoul, Korea. Her research focuses on effects of new media technologies on users and society.

Matthew P. McAllister

is Professor of Communications in the Department of Film/Video \& Media Studies at The Pennsylvania State University, USA. He is the co-editor of The Advertising and Consumer Culture Reader (2009, Routledge, with Joseph Turow), and The Routledge Companion to Advertising and Promotional Culture (forthcoming, Routledge, with Emily West). 